\title{
Doralice Fabiano, Senza Paradiso. Miti e credenze
} sull'aldilà greco

(Collana del Centro di Antropologia del Mondo Antico dell'Università di Siena), Il Mulino, Bologna, 2019, pp. 278, ISBN: 978-88-15-27996-5, $20 €$.

\section{Nicola Cusumano}

\section{(2) OpenEdition}

Journals

\section{Edizione digitale}

URL: https://journals.openedition.org/mythos/2623

DOI: $10.4000 /$ mythos. 2623

ISSN: 2037-7746

Editore

Salvatore Sciascia Editore

Notizia bibliografica digitale

Nicola Cusumano, «Doralice Fabiano, Senza Paradiso. Miti e credenze sull'aldilà greco», Mythos [Online], 14 | 2020, online dal 31 décembre 2020, consultato il 05 octobre 2021. URL: http://

journals.openedition.org/mythos/2623; DOI: https://doi.org/10.4000/mythos.2623

Questo documento è stato generato automaticamente il 5 octobre 2021.

Mythos 


\section{Doralice Fabiano, Senza Paradiso. Miti e credenze sull'aldilà greco}

(Collana del Centro di Antropologia del Mondo Antico dell'Università di Siena), Il Mulino, Bologna, 2019, pp. 278, ISBN: 978-88-15-27996-5, $20 €$.

\section{Nicola Cusumano}

1 Dedicato al mondo dei defunti e alle credenze che, nel mondo greco, gli danno forma e struttura (con particolare attenzione nell'ultima parte alle punizioni oltremondane, finora piuttosto trascurate nel dibattito specialistico recente), il libro di Doralice Fabiano si presenta al lettore sotto il segno di un forte impegno metodologico e di una sensibilità storico-culturale che percorre tutto il piano dell'opera. Due prospettive di ricerca sono richiamate in queste pagine, quella dell'antropologia storica e quella della storia delle religioni: la loro intersezione, come esplicitamente dichiara l'Autrice, mira a far reagire la prospettiva emic (le categorie di pensiero elaborate all'interno dell'oggetto di ricerca, in questo caso nella cultura greca) con quella etic (le categorie di pensiero elaborate dagli studiosi per interpretare i dati culturali oggetto di studio). Muoversi tra queste due opzioni aiuta a vigilare sulle insidie che incombono in ogni esperienza conoscitiva, soprattutto perché i codici culturali con cui ci orientiamo nel mondo sono oggetti storici esposti all'erosione e al mutamento. In particolare, come sottolinea D. Fabiano, per i Greci (e in generale a proposito delle società 'antiche') il rapporto con gli dei non è centrato "sulla promessa di una vita e di una salvezza ultraterrena in un mondo "altro»", ma su una pratica rituale quotidiana e terrena che rinforza e mantiene l'ordine stabilito dagli dèi e garantisce in tal modo il benessere della comunità umana (p. 9). Perciò non si dovrebbe commettere l'errore di attribuire un ruolo cruciale a questo aspetto, contrariamente a quanto avviene nei monoteismi: la religione greca è "senza paradiso (e senza inferno!)". La storia delle religioni, per la sua natura di disciplina epistemologicamente aperta e a cavallo di diverse sensibilità metodologiche, è attrezzata per supportare questa sorta di 'vigilanza epistemologica', che si traduce in una rimessa in discussione continua e attenta a mettere a nudo l'incessante tensione tra emico ed etico che caratterizza la ricerca nel campo delle scienze umane. 
2 Il volume si divide in cinque capitoli, a loro volta distribuiti in due parti. La prima (Rappresentare l'invisibile, 15-124) introduce l'argomento del libro, le rappresentazioni del mondo dei morti in Grecia, attraverso un itinerario tematico: l'aspetto dei defunti, il nutrimento loro riservato e la strutturazione dello spazio in cui sono collocati una volta oltrepassato il mondo dei viventi. Il primo dei tre capitoli che compongono questa sezione (Dare corpo alle ombre, 17-44) dà conto dei modi in cui i Greci hanno immaginato la condizione del defunto, a iniziare dalla sua rappresentazione, nell'intento di dare conto della "complessa costruzione culturale della corporeità del defunto" (p. 45): poiché di tale condizione non vi può essere esperienza diretta in vita, ogni elemento relativo ai modi di questa rappresentazione oltremondana "rivela di volta in volta le associazioni simboliche che questo mondo suscita all'interno di un determinato quadro culturale" (p. 17). È la categoria concettuale di "corpo sostitutivo" a guidare l'analisi delle rappresentazioni greche dei defunti in questo primo capitolo. La crisi transizionale che si manifesta nell'intervallo tra la morte e la sepoltura trova soluzione nell'azione rituale svolta dal gruppo di appartenenza, che così ha in questo delicato e traumatico momento di passaggio l'occasione di mettere in scena e consolidare la rete di legami familiari e sociali e al tempo stesso rimarginare la lacerazione inferta dalla morte: ciò spiega bene la dimensione collettiva del lutto, attraverso cui passa l'identità sociale e l'affermazione dei legami familiari (p.28). Oltre al cadavere sepolto nella tomba vi è anche la psyche, la forma con cui i defunti giungono nell'aldilà. Ma, avverte l'A., non bisogna farsi ingannare dalla nostra familiarità con questo termine (ecco qui un esempio di possibile cortocircuito tra emic ed etic): non di anima si tratta, nel senso da noi attribuito al termine, ma di ciò che "sostituisce l'individuo nei momenti in cui egli non ha più controllo e percezione di se stesso, quali il sonno, la trance, la morte" (p. 29). È chiaro che sono qui in gioco due differenti concezioni della 'persona', la nostra e quella che agisce in Omero, anzi è tutta la struttura psicologica dell'individuo ad essere molto diversa dalla nostra. D'altronde, la nostra nozione di 'persona' resta senza un esatto corrispondente nella cultura greca. L'analisi è poi estesa ad altri corpi sostitutivi del defunto, come eidolon e skia: per tutti vale la constatazione che, in condizioni ordinarie, tali 'corpi' restano al di fuori del campo percettivo dei vivi (p. 40); possono però in certi casi (si tratta dei morti di morte violenta e rimasti insepolti) provocare apparizioni e fenomeni sonori inquietanti, generare terrore e introdurre così tra i vivi disordine e paura: questi fenomeni però, pur generati da eventi violenti che hanno portato alla morte, non sono pure e semplici apparizioni del defunto, ma entità per lo più collettive prive di un'identità personale che perseguitano il colpevole. Nel secondo capitolo (Il cibo dei morti, 45-85) è sviluppato il tema delle offerte alimentari per i defunti come strumento di mantenimento del rapporti tra morti e vivi (che però mai si devono mescolare tra loro) nel più ampio quadro delle molteplici funzioni sociali assegnate ai defunti: offrire regolarmente cibo e libagioni ai defunti costituisce "il cuore del culto tributato al morto" (p.52). Quello che conta in questo genere di relazioni è la logica 'esclusiva' della separazione che deve contraddistinguere il rapporto tra morti e vivi: il cibo dei morti è "una forma estrema di cibo degli «altri»" (p. 58), nel senso che si tratta di cibi e bevande dotati di forte anomalia rispetto al codice alimentare dei viventi (per es. vino puro o latte e miele). Che i morti possano essere ospiti davvero problematici, lo dimostra il caso degli Anthesteria, che nel corso della sua seconda tappa (i Choes) prevede il temporaneo ritorno dei morti nella polis e impone perciò ai cittadini l'adozione di particolari rimedi profilattici (ma l'A. non manca di sottolineare la problematicità di questa interpretazione in un lemma del 
Lexicon di Fozio). Al tema del rischio derivante dal contatto tra morti e vivi a seguito di un atto violento, in particolare l'omicidio, e alle procedure che implicano cibo e ospitalità per disattivare la minaccia sono rivolte anche le pagine dedicate alle c.d. lex sacra di Selinunte e lex cathartica di Cirene: il principio fondamentale all'opera, qui come nelle sezioni successive, è quello per cui "i defunti non possono condividere la stessa tavola dei viventi” (p. 81). Nel terzo capitolo (L'isola che non c’è, 87-124) la categoria concettuale di 'corpo sostitutivo', già vista efficacemente applicata alla rappresentazione del defunto e delle dinamiche che gli sono attribuite, entra in gioco anche per la rappresentazione dello spazio in cui dimorano i defunti. Si tratta di uno spazio che la documentazione declina al plurale e che perciò non va identificato unicamente con il regno di Hades, ma con una serie di 'luoghi' che hanno in comune con quelli dei vivi alcuni elementi di riconoscibilità, sia pure all'insegna della estraneità e inospitalità: oscurità, isolamento, natura acquatica, nebbia, marginalità etc. In quanto spazio chiuso e separato da quello dei viventi, il mondo dei morti è collocato ai confini del mondo, in senso orizzontale (Isola dei Beati) o lungo un asse verticale (Hades, Tartaro). Come osserva l'A., siamo di fronte ad "una rappresentazione geografica dinamica" (p. 95) che soddisfa, di volta in volta, la struttura narrativa che ne costituisce il contesto. Nel caso dell'Hades colpisce la "concezione «architetturale»" (p. 106) della dimora dei morti, strutturata nei suoi vari elementi come una casa il cui dinamismo funziona però in una sola direzione, dall'esterno verso l'interno e mai viceversa: si può accedere ma non si può più uscirne. Allo spazio dei morti la tradizione assegna un ricco sistema idrografico (fiumi, paludi, fonti) che innerva quel mondo stabilendo connessioni tra diversi piani cosmici: le rappresentazioni di Acheronte, Stige, Piriflegetonte e Cocito sono analizzate nelle loro diverse peculiarità e nei diversi modi di costituirsi al tempo stesso come confine, passaggio e separazione: anche in questo caso tanto in orizzontale (Acheronte) che in verticale (Stige). Filo comune che riunisce e ordina una geografia così complessa è il carattere fortemente disorientante delle linee di confine tra morti e vivi: è così ribadita una differenza evidente tra i due mondi, quello ordinato dei vivi e percorso da itinerari riconoscibili e stabili, e quello dei defunti che a tale riconoscibilità e stabilità si sottrae, tanto da poter parlare di una "dimensione a-direzionale in cui il defunto corre il rischio di vagare senza meta" (p.120). Una conferma illuminante può essere individuata nelle istruzioni di viaggio rivolte ai defunti nelle lamine orfiche. La spersonalizzazione così ottenuta della rappresentazione dell'aldilà avvicina, secondo il suggerimento dell'A., questo spazio ai 'non-luoghi' oggetto di studio di M. Augé, "cioè come uno spazio che esige da chi vi entra di accettare la perdita della propria identità fisica e sociale e al tempo stesso nega ogni organizzazione degli individui in forma di società" (p. 124).

Come annunciato nell'introduzione, la seconda parte del volume (Punizioni infere, 125-240) si concentra sulla sorte dei defunti, con particolare attenzione alle punizioni cui possono andare incontro nell'aldilà, tenendo tuttavia conto che tali punizioni non implicano la categoria escatologica di 'giudizio' presente nei monoteismi storici. Mettere in luce questa distanza, ricorrendo di nuovo fruttuosamente alla tensione tra etic ed emic, è l'obiettivo del primo dei due capitoli in cui si articola questa seconda parte (Un giudizio non universale, 127-165). Leggendo tra le pieghe di un passo plutarcheo (I ritardi della punizione divina 18, 560 F-561 A), l'A. enuclea tre elementi di riflessione che aiutano il lettore a fare i conti con la distanza che caratterizza codici culturali diversi: a) la presenza di modelli alternativi a quello giudiziario (per es., quello agonisticosportivo) per esprimere l'idea di giustizia retributiva e quindi di punizione 
oltremondana; b) il problema della credibilità che coglie il peculiare atteggiamento greco nei confronti di tali punizioni e punta piuttosto su un'idea di retribuzione che si manifesta in punizioni inflitte ai discendenti dei colpevoli o addirittura alla loro polis; c) infine, la natura della giustizia esercitata dagli dei sui vivi ha un tratto in comune con quella umana, cioè "esercitare un potere deterrente nei confronti di coloro che hanno intenzione di compiere azioni malvagie" (p. 130): questo rende il tema delle punizioni oltremondane non solo problematico ma anche particolarmente adatto a illuminare lo scarto, in termini di rappresentazione e di ideologia, che ci separa da quella cultura. Il punto è che la religione della polis non ha come obiettivo "una qualche forma di «salvezza» dell'individuo in un mondo «altro» dal nostro e trascendente, ma quello di creare e garantire l'equilibrio della relazione tra mortali e immortali nella dimensione terrena" (p. 130). I racconti sulle punizioni 'infere' somministrate a celebri personaggi del mito vanno allora inquadrati in un diverso paradigma di verità rispetto al nostro, come ha messo in evidenza Paul Veyne. Una traccia è riconosciuta dall'A. in alcune celebri sezioni della Repubblica e del Fedone di Platone: il loro esame, che ruota intorno al concetto di 'fiducia' (pistis, intesa in senso attivo) e alla sua assenza (apistia), consente di avvicinarsi ad una "corretta comprensione delle categorie emiche" qui all'opera (p.135), che delineano un contesto culturale in cui l'azione della pistis produce credenza, ma non la condizione di "«aver fede» nel senso moderno del termine" (p.139). In altre parole, è una credenza che non dà luogo ad un dogma, come l'intendiamo noi. Va però sottolineato che il 'destino' speciale che attende il defunto nell'aldilà può dipendere anche da scelte compiute in vita in cui non entra in gioco il modello giudiziario. Particolare rilevanza hanno le iniziazioni legate a divinità come Dioniso, Demetra e Kore, che hanno la capacità di intervenire nello spazio dell'Hades, assicurando al defunto "uno speciale trattamento dopo la morte" (p. 141). In particolari contesti iniziatici, tale trattamento è assicurato dal rispetto di certe istruzioni che accompagnano il cammino del defunto fino alla sua dimora finale e che "gli consentiranno di sfuggire alla sorte dei comuni mortali" (p.145). È il caso delle laminette c.d. orfiche e la loro rappresentazione del paesaggio oltremondano e delle prove cui il defunto si sottomette per essere ammesso in una speciale comunità privilegiata, obiettivo per il quale si è già preparato in vita tramite i rituali iniziatici. Si delinea così la possibilità per alcuni dei defunti di accedere ad una condizione privilegiata da cui gli altri restano esclusi. Quanto fin qui osservato non deve però farci escludere del tutto il ruolo del modello giudiziario applicato alla sorte degli uomini nell'aldilà. Lo sottolinea l'A. servendosi con acume di due passaggi eschilei, nelle Supplici e nelle Eumenidi. Anche Platone fornisce, nel Gorgia e nel Fedone, illuminanti elementi di riflessione a riguardo dei miti inferi di punizione: qui le colpe commesse in vita sono giudicate e sottoposte a punizioni che richiamano la logica giudiziaria codificata ad Atene. La giustizia tra i morti, specularmente a quella amministrata dai vivi, risponde ad una simile esigenza al tempo stesso punitiva e correttiva (come l'A. rileva in alcuni altri passaggi platonici): il beneficio della punizione è di regola direttamente proporzionale alla sua pubblica spettacolarizzazione, tra i vivi come tra $i$ morti: un aspetto che rimanda in filigrana alla riflessione antica sulla finalità prospettica della punizione. Il quinto e ultimo capitolo (Delitti e castighi, 167-240) si concentra infine su alcune famose punizioni infere, a cominciare da quelle descritte nella Nekyia omerica: Tizio, Tantalo e Sisifo. Il loro schema si basa sulla reiterazione all'infinito della pena e sulla frustrazione indotta dall'impossibilità di completare l'azione prevista (nei casi di Tantalo e Sisifo bere e mangiare oppure spingere su 
un'altura un masso che ricade giù in prossimità del traguardo; nel caso di Tizio si tratta di un dolore rinnovato all'infinito e rappresentato dal fegato eternamente divorato da due avvoltoi): uno schema che si ripete anche nella maggior parte degli altri racconti di punizioni infere. Un aspetto che colpisce il lettore è la scarsa o quasi nulla informazione sulle colpe commesse, quindi sulla relazione di merito con la punizione. L'interpretazione proposta dall'A. va cercata nella cornice narrativa delle peripezie di Odisseo e delle motivazioni che lo spingono all'esperienza della Nekyia: si tratta di exempla destinati ad esercitare un "potere deterrente" (p. 173) tanto sul protagonista Odisseo che sull'uditorio intorno alla "impossibilità a sfuggire alla collera degli dèi e sul carattere implacabile di questa violenta ira, che colpisce persino nell'aldilà, ben oltre l'estensione della vita umana" in questo e nell'altro mondo. L'altro elemento messo convincentemente in luce è il sentimento di frustrazione per la condizione di impotenza che sembra costituire il nucleo principale di queste punizioni: si tratta, come sottolinea l'A., di "uno dei peggiori incubi di una società in cui la rivalità e il desiderio di primeggiare (aristeuein) sono al centro della costruzione delle relazioni umane" (p. 178). La bella analisi della storia di Sisifo trasmessa in un frammento di Ferecide consente all'A. di riportare la logica della sua punizione al tentativo di disarticolare i confini tra mondo dei vivi e dei morti, annullando con l'astuzia una separazione che deve restare insuperabile. La pietra continuamente spinta da Sisifo rinvia all'horos, la pietra di confine che appunto separa tra i vivi le proprietà e i confini tra stati, e in questa storia tiene divisi i due piani cosmici. Sisifo ha invece violato il confine, che come è noto consente ai defunti di entrare, ma non di uscire: spingere verso l'alto il masso ma essere continuamente risospinto in basso rinvia al divieto di compiere il tragitto in senso vietato: l'aldilà è un "mondo che blocca e imprigiona chi vi entra" (p. 209). Altrettanto puntuale e penetrante l'analisi, che occupa l'ultima parte del volume, dedicata alle condizioni punitive che attendono dopo la morte i non iniziati condannati a svolgere attività frustranti e senza senso, come "riempire d'acqua una giara forata e attingere acqua con un setaccio" (p. 213).

Più ricco di contenuti e riflessioni di quanto possa registrare questa recensione, il volume di Doralice Fabiano è il frutto di una ricerca rigorosa che, grazie al linguaggio scorrevole e mai accademico (ma non per questo corrivo), si rivolge anche ad un pubblico non specialista. La premessa metodologica è coerentemente attiva lungo tutto lo sviluppo dell'indagine: la ricca documentazione è puntualmente passata al vaglio di tale premessa e continuamente messa alla prova. Il lettore (accademico e non) ne apprezzerà anche la densità concettuale che sostiene uno sviluppo tematico di grande ampiezza: la massa di dati qui discussa non appesantisce la lettura ma al contrario la sostiene persuasivamente ancorandola alla concretezza dei documenti di volta in volta proposti all'occhio del lettore. D'altra parte il lungo elenco di riferimenti bibliografici è spesso occasione per osservazioni critiche sulla storiografia moderna. 


\section{AUTORI}

NICOLA CUSUMANO

Università di Palermo

Dipartimento Culture e Società

Edificio 15, Viale delle Scienze

90128 Palermo

e-mail nicola.cusumano25(at)unipa.it 